\title{
Short stature in a girl with partial monosomy of the pseudoautosomal region distal to DXYS15: further evidence for the assignment of the critical region for a pseudoautosomal growth gene(s)
}

Tsutomu Ogata, Atsuko Yoshizawa, Koji Muroya, Nobutake Matsuo, Yoshimitsu Fukushima, Gudrun Rappold, Susumu Yokoya

\begin{abstract}
This report describes a 12 year 10 month old girl with short stature and a non-mosaic $46, X, X p+$ karyotype. Her height remained below -2 SD of the mean, and her predicted adult height $(143 \mathrm{~cm})$ was below her target height $(155.5 \mathrm{~cm})$ and target range $(147.5 \mathrm{~cm}-163.5 \mathrm{~cm})$. Cytogenetic and molecular studies showed that the $\mathbf{X p}+$ chromosome was formed by an inverted duplication of the Xp21.3-Xp22.33 segment and was missing about $700 \mathrm{~kb}$ of DNA from the pseudoautosomal region distal to DXYS15. The results provide further support for the previously proposed hypothesis that the region between DXYS20 and DXYS15 is the critical region for a pseudoautosomal growth gene(s).
\end{abstract}

( $\mathcal{F}$ Med Genet 1995;32:831-834)

Although genotype-phenotype correlations in patients with partial monosomy of the pseudoautosomal region (PAR) suggest that the region between DXYS20 and DXYS15 is the critical region for a pseudoautosomal growth gene(s) (P growth gene (s)), ${ }^{1}$ the assignment is primarily based on the report of a single patient with short stature and a terminal deletion of the PAR distal to DXYS $15 .^{2}$ Here, we describe a Japanese girl with short stature and a rearranged $\mathrm{X}$ chromosome. Genotype-phenotype correlation of this girl provides further support for the assignment of the critical region for the $P$ growth gene(s).

This girl was the $48.0 \mathrm{~cm}$ (mean $-0.8 \mathrm{SD}$ ) and $2 \cdot 2 \mathrm{~kg}$ (mean $-2 \cdot 2 \mathrm{SD}$ ) product of an uncomplicated term pregnancy. Her postnatal course was uneventful, and menarche occurred at 11 years 3 months of age. At 11 years 8 months, she was seen at Toranomon Hospital because of short stature. Physical examination showed no abnormality except for proportionate short stature. Secondary sexual development was appropriate for her age (Tanner stage: breast 4, pubic hair 3). Mental development appeared normal. Routine laboratory tests were normal, as were endocrine and radiological studies for short stature. At present, she is 12 years 10 months old and has regular menses.

The growth chart of the patient is shown in fig 1 . Her height remained below $-2 \mathrm{SD}$ of the mean height of normal Japanese girls. ${ }^{3}$ Furthermore, her predicted adult height $(\mathrm{PAH}$, a child's final height as predicted from biological data of the child) was below her target height (TH, a child's final height as predicted from the parental height) and target range (TR, $95 \%$ confidence interval of $\mathrm{TH})$. PAH was determined as $143 \mathrm{~cm}$ (mean $-2.7 \mathrm{SD}$ ) by the method of Ito and Yokoya, ${ }^{4}$ and TH and TR were obtained as $155.5 \mathrm{~cm}$ (mean $-0.3 \mathrm{SD}$ ) and $147.5 \mathrm{~cm}-163.5 \mathrm{~cm}$ (mean $-1.8 \mathrm{SD}$ mean $+1 \cdot 2 \mathrm{SD}$ ), respectively, by the equations of Ogata et al. ${ }^{5}$

The patient's karyotype was $46, \mathrm{X}, \mathrm{Xp}+$ in all the 263 lymphocytes analysed. High resolution $\mathrm{G}$ banding ${ }^{6}$ indicated that the $\mathrm{Xp}+$ chromosome was formed by an inverted duplication of the Xp21.3-Xp22.33 segment (fig 2 ). $R$ banding $(R B G)^{7}$ showed that the $X p+$ chromosome was late replicating in 76 of 101 lymphocytes examined and the normal X chromosome was late replicating in the remaining 25 lymphocytes. Fluorescence in situ hybridisation analysis using an $\mathrm{X}$ chromosome specific cocktail mixture of probes covering the entire $\mathrm{X}$ chromosome ${ }^{8}$ detected positive signals on the entire $\mathrm{Xp}+$ chromosome. The parents had a normal karyotype.

To determine whether the $\mathrm{Xp}+$ chromosome was associated with a deletion of the PAR, Southern blot analysis was performed on the patient, the mother, and normal controls. Representative results are shown in fig 3 and summarised in the table. Informative restriction fragment length polymorphism was obtained only for DXYS 14; maternal bands for DXYS14 were not inherited by the patient, implying that the patient's DXYS14 was of paternal origin 


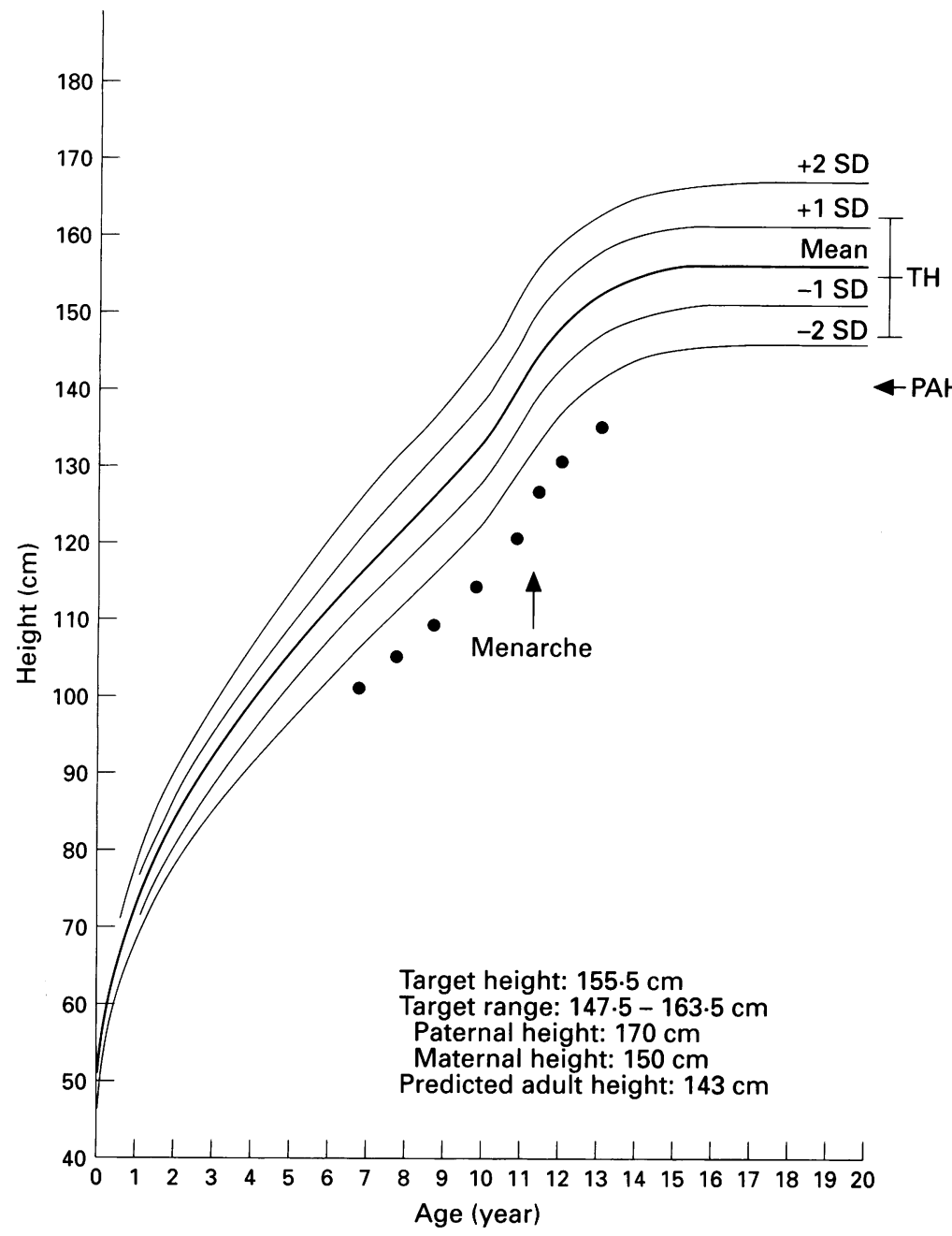

Figure 1 Growth chart of the patient. The dots represent the actual heights of the patient. The growth curves depict the longitudinal height standards for normal fapanese girls. Target height (TH) indicates a child's final height as predicted from the parental height, target range indicates $95 \%$ confidence interval of $T H$, and predicted adult height (PAH) indicates a child's final height as predicted from biological data of the child.

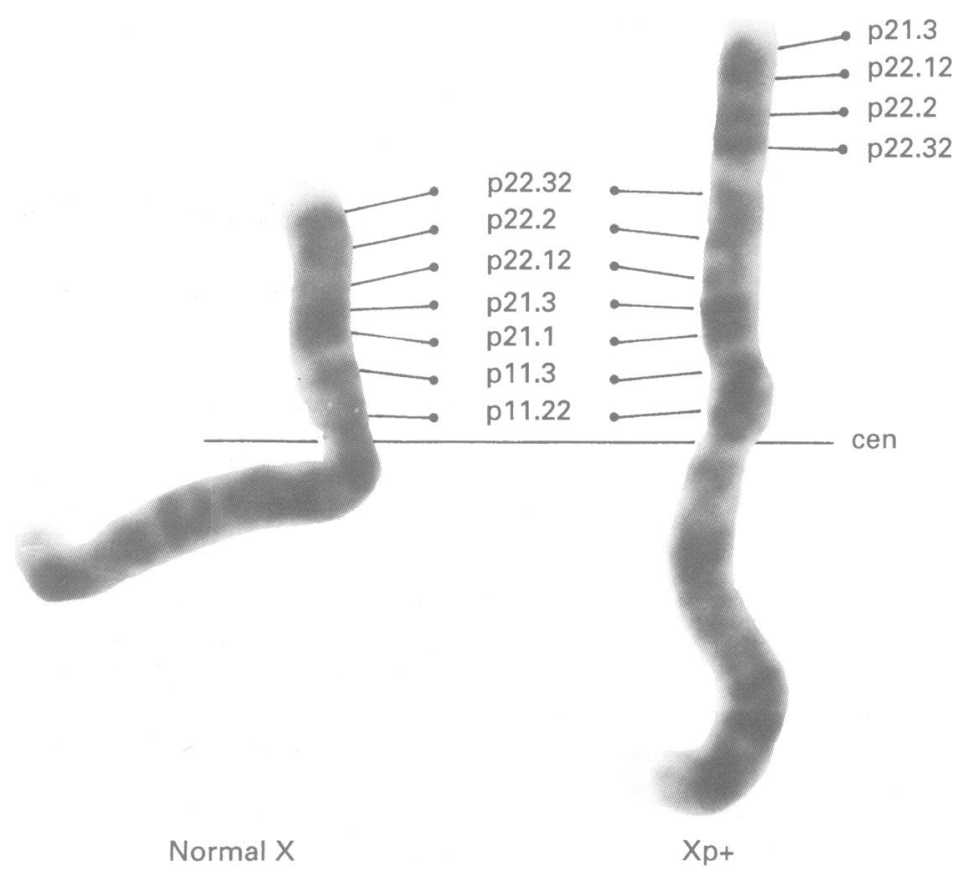

Figure 2 The normal $X$ chromosome (left) and the $X p+$ chromosome (right) of the patient. The band pattern of the extra chromosomal material is consistent with an patient. The band pattern of the extra chromosomal materi
inverted duplication of the $X p 21.3-X p 22.33$ segment. and present in a single copy. The comparison of band intensity using the band for autosomal TK gene as an internal control indicated that, in the patient, DXYS60, DXYS87, DXYS161, DXYS28, and DXYS59 were present in a single copy, whereas DXYS15, CSF2RA, DXYS17, and MIC2 were present in triple copies.

The results indicate that the $\mathrm{Xp}+$ chromosome was formed by an inverted duplication of the Xp21.3-Xp22.33 segment and was missing the distal part of the PAR. It is inferred that the $\mathrm{Xp}+$ chromosome was generated during maternal meiosis (fig 4). Sine the Xp + chromosome was the sole discernible abnormality, it is probable that the short stature of our patient was caused by the $\mathrm{Xp}+$ chromosome.

The most pertinent explanation for the short stature would be to postulate that the $\mathrm{P}$ growth gene(s) is deleted or disrupted in our patient. In this context, it is noteworthy that the $\mathrm{Xp}+$ chromosome is missing only about $700 \mathrm{~kb}$ of DNA from the PAR distal to DXYS15 (table). The result is consistent with the notion that the region from DXYS20 to DXYS15 is the critical region for the $\mathrm{P}$ growth gene(s). ${ }^{1}$ Furthermore, the difference between PAH and TH of our patient is in close agreement with the adult height difference between 13 females with $46, \mathrm{X}, \operatorname{del}(\mathrm{X})(\mathrm{p} 22.32)$ and nine females with 46 , $\mathrm{XX}$ in the two pedigrees reported by Curry et $a l^{13}(154 \cdot 1$ (SD 4.9) $\mathrm{cm} v 166.3$ (SD 4.0$) \mathrm{cm}$, $\left.\mathrm{p}<10^{-5}\right)$. This would also be compatible with the $\mathrm{P}$ growth gene(s) being impaired in our patient. Although it might be possible that the $\mathrm{P}$ growth gene(s) is preserved on the $\mathrm{Xp}+$ chromosome but is subject to a position effect or to an abnormal spreading of $\mathrm{X}$ inactivation, as has been suspected for large $\mathrm{Xq}-$ chromosomes, ${ }^{14}$ the possibility remains purely speculative at present.

By contrast, other factors relevant to the $\mathrm{Xp}+$ chromosome are unlikely to explain the short stature adequately. First, several genes on the duplicated segment could affect stature because of the dosage effect. In this regard, genes escaping $\mathrm{X}$ inactivation would be present in three active copies in all somatic cells, and genes normally subject to $\mathrm{X}$ inactivation would be present in two active copies in somatic cells with the active $\mathrm{Xp}+$ chromosome. However, height comparison between white patients with 45, X (143.3 (SD 6.4) cm, n=130), 46, XX $(162 \cdot 2(\mathrm{SD} 6 \cdot 0) \mathrm{cm}$, the British standard), and $47, \mathrm{XXX}(167.9(\mathrm{SD} 7 \cdot 7) \mathrm{cm}, \mathrm{n}=19)^{15}$ argues against the $\mathrm{X}$ chromosome carrying a gene(s) escaping $\mathrm{X}$ inactivation that has a growth suppressing effect, and there is no evidence for the presence of a gene(s) subject to $\mathrm{X}$ inactivation that acts as a growth suppressor. Second, chromosome imbalance would cause global non-specific developmental defects, exerting a disadvantageous effect on statural growth. ${ }^{15}$ However, it appears difficult to ascribe the short stature to chromosome imbalance alone. (1) If the $\mathrm{P}$ growth gene(s) is preserved on the $\mathrm{Xp}+$ chromosome, it would be present in three copies, and 47,XXX patients with three copies of the $\mathrm{P}$ growth gene(s) are taller than normal females. ${ }^{15}$ Thus, unless the $P$ growth gene(s) is impaired, somatic cells of our patient, at 
The copy number of pseudoautosomal loci analysed in the present study

\begin{tabular}{|c|c|c|c|c|c|c|c|}
\hline Locus (probe) & Distance from telomere $(k b)$ & Enzyme & Patient & Mother & Male & Female & Reference \\
\hline $\begin{array}{l}\text { DXYS14 (29C1) } \\
\text { DXYS60 (U7A) } \\
\text { DXYS87 (P99) } \\
\text { DXYS161 (B6) } \\
\text { DXYS28 (pDP411) } \\
\text { DXYS59 (68A) } \\
\text { DXYS15 (113D) } \\
\text { CSF2RA (cDNA) } \\
\text { DXYS17 (601) } \\
\text { MIC2 (19B) }\end{array}$ & $\begin{array}{l}<20 \\
310-360 \\
380-420 \\
470-476 \\
482-486 \\
500-650 \\
700-760 \\
1180-1300 \\
1840-1880 \\
2500-2600\end{array}$ & $\begin{array}{l}\text { TaqI } \\
\text { EcoRI } \\
\text { TaqI } \\
\text { Eco RI } \\
\text { Eco RI } \\
\text { TaqI } \\
\text { TaqI } \\
\text { EcoRI } \\
\text { Eco RI } \\
\text { TaqI }\end{array}$ & $\begin{array}{l}1 \\
1(0.37) \\
1(1 \cdot 15) \\
1(0 \cdot 48) \\
1(0.58) \\
1(1.49) \\
3(2 \cdot 32) \\
3(0.68) \\
3(0.98) \\
3(3.63)\end{array}$ & $\begin{array}{l}2 \\
2(0.75) \\
2(2 \cdot 08) \\
2(0.81) \\
2(0.91) \\
2(2.83) \\
2(1.45) \\
2(0.47) \\
2(0.63) \\
2(2.50)\end{array}$ & $\begin{array}{l}2 \\
2(0.78) \\
2(1.97) \\
2(0.78) \\
2(0.88) \\
2(2.98) \\
2(1.31) \\
2(0.48) \\
2(0.75) \\
2(2.81)\end{array}$ & $\begin{array}{l}2 \\
2(0.68) \\
2(1.89) \\
2(0.76) \\
2(0.85) \\
2(2.85) \\
2(1.37) \\
2(0.45) \\
2(0.60) \\
2(2.35)\end{array}$ & $\begin{array}{r}9 \\
10 \\
10 \\
10 \\
10 \\
11 \\
10 \\
10 \\
10 \\
10\end{array}$ \\
\hline
\end{tabular}

The copy number of DXYS14 is determined by the RFLP pattern (see fig 3), and that of the remaining loci is based on the band intensity analysis using a laser densitometer (ACD-250DX, ATTO). The values in parentheses represent the ratio of the band intensity between each locus and autosomal TK gene. ${ }^{12}$ Simila band intensity ratios have been reproduced by other enzyme-probe combinations (data not shown). The positions of the pseudoautosomal loci are based on the reports of Henke et al ${ }^{10}$ and Petit et al. ${ }^{11}$

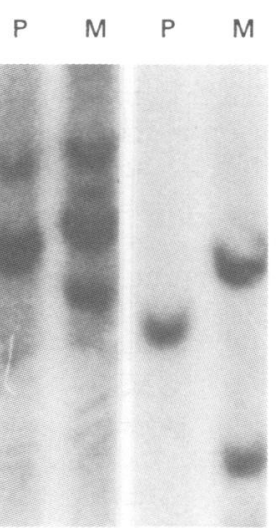

[1]

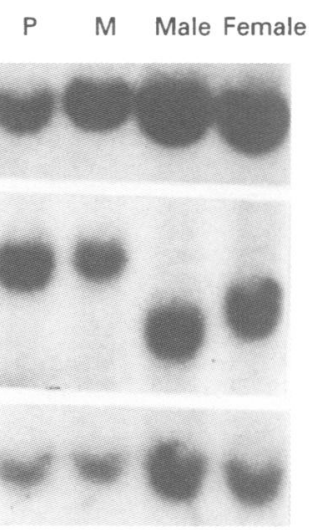

[2]

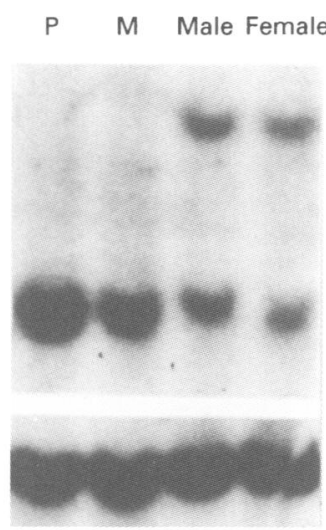

[3]

Figure 3 Southern blot analysis ( $P=$ patient; $M=$ mother, normal male, and normal female). (1) EcoRI digests (left) and TaqI digests (right) hybridised with 29C1 (DXYS14). Maternal bands are not inherited by the patient. (2) TaqI digests hybridised with $68 A$ (DXYS59, top panel), 113D (DXYS15, middle panel), and the probe for autosomal TK gene (bottom panel) (same filter). Although comparison of the band intensity for the TK gene indicates that the DNA loaded is similar between the patient and the mother, the band intensity for DXYS59 is weaker in the patient than in the mother and that for DXYS15 is stronger in the patient than in the mother. The band intensity pattern for the three loci is similar between the mother and the normal subjects. (3) EcoRI digests hybridised with 601 (DXYS17, upper panel) and the probe for autosomal TK gene (lower panel) (same filter). Although comparison of the band intensity for TK gene implies that the DNA loaded is similar between the four subjects, the band intensity for DXYS17 is stronger in the patient that in the mother and the normal subjects.
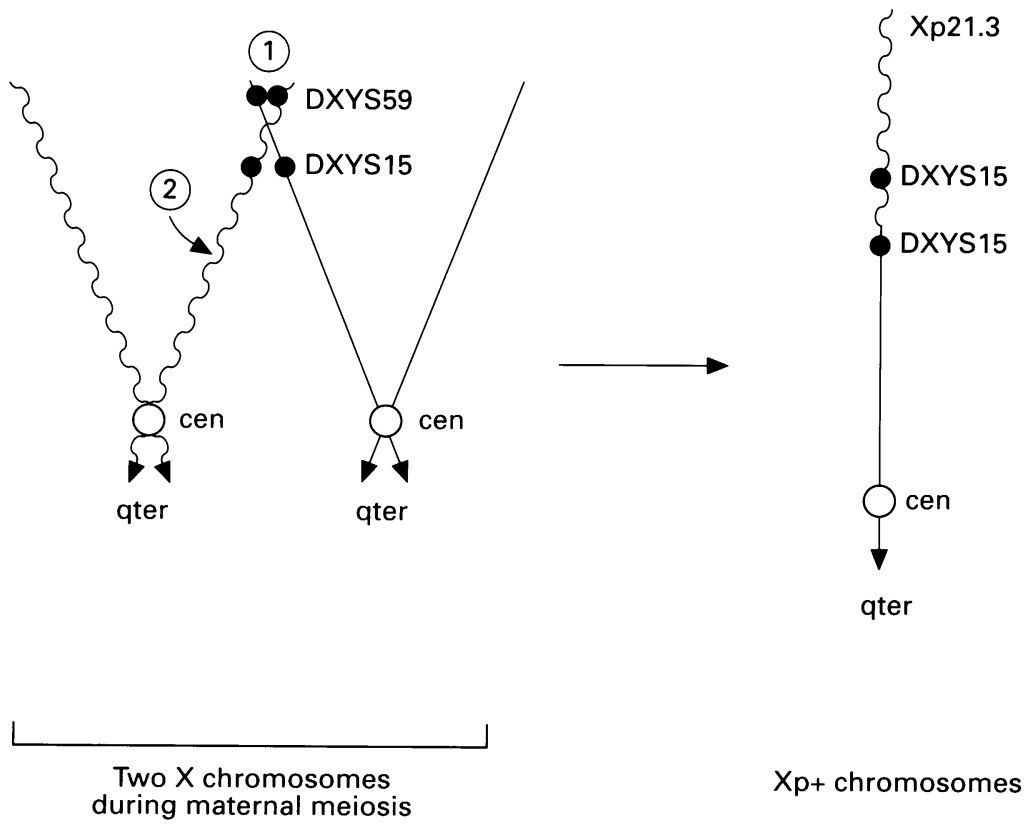

$\mathrm{Xp}+$ chromosomes

Figure 4 A schematic representation of the generation of the Xp+chromosome. It is inferred that crossing over btween DXYS59 and DXYS15 (a normal event designated by the number 1) and chromosomal breakage at Xp21.3 (an abnormal event designated by the number 2) occurred during maternal meiosis and, subsequently, the distal Xp segmen between the crossing over point and the chromosomal breakpoint was transferred onto the homologous $X$ chromosome, resulting in the inverted duplication of the Xp21.3-Xp22.33 segment and in the deletion of the telomeric portion distal to DXYS15.

least those with the inactive $\mathrm{Xp}+$ chromosome, would have sufficient growth potential. (2) Although chromosome imbalance would be severer in somatic cells with the active $\mathrm{Xp}+$ chromosome than in those with the inactive $\mathrm{Xp}+$ chromosome, the $\mathrm{X}$ inactivation pattern suggests that the deleterious effects of chromosome imbalance as well as those of perturbed gene dosage are not so different between the two types of cells. (3) Our patient lacked non-specific features attributable to chromosome imbalance such as mental retardation and somatic anomalies. This agrees with the notion that clinical effects caused by chromosomal duplication are much milder than those caused by corresponding chromosomal deletions, ${ }^{1516}$ and implies that the phenotypic effects of chromosome imbalance remained at mild or subclinical level in our patient. Third, if the $\mathrm{Xp}+$ chromosome is inactivated preferentially, this implies the operation of a mild degree of cell selection mechanism that could cause growth failure by reducing the total number of viable cells in a person. ${ }^{17}$ In this connection, since the inactivation pattern of lymphocytes does not necessarily reflect that of other tissues, several tissues may be associated with a very skewed inactivation pattern. However, normal adult height in white patients with $46, \mathrm{X}, \mathrm{t}$ (X;autosome) accompanied by non-random inactivation of the normal $\mathrm{X}$ chromosome $(161.6(\mathrm{SD} 7 \cdot 6) \mathrm{cm}, \mathrm{n}=17)^{15}$ argues that the cell selection mechanism, if it occurs, does not cause growth failure.

We would like to thank Dr Jun Kusuda for the band intensity analysis, and Dr Christine Petit for probes U7A and 68A. This work was supported in part by a grant for Paediatric Research from the Ministry of Health and Welfare, a grant from Vehicle Racing Commemorative Foundation, and a grant from Keio University.

1 Ogata T, Petit C, Rappold G, Matsuo N, Matsumoto T, Goodfellow P. Chromosomal localisation of a pseudoautosomal growth gene(s). 7 Med Genet 1992;29:624-8.

2 Ogata T, Goodfellow P, Petit C, Aya M, Matsuo N. Short stature in a girl with a terminal $\mathrm{Xp}$ deletion distal to DXYS15: localisation of a growth gene(s) in the pseudoautosomal region. 7 Med Genet 1992;29:455-9.

3 Suwa S, Tachibana K, Maesaka H, Tanaka T, Yokoya S. Longitudinal standards for height and height velocity for Japanese children from birth to maturity. Clin Pediatr Endocrinol 1992;1:5-13.

4 Ito R, Yokoya S. Predicting adult height from skeletal age: a modified Bayley-Pinneau method for Japanese children. Annual Report of fapan Athletic Association 1994; No 6: A6-53.

5 Ogata T, Matsuo N, Tamai S, Osano M, Tango T. Target height and target range for the Japanese. Fpn f P Paediatr
$1990 ; 94: 1535-40$.

6 Ikeuchi T. Inhibitory effect of ethidium bromide on mitotic chromosome condensation and its application to highresolution chromosome banding. Cytogenet Cell Genet 1984

7 Wolf S, Perry P. Differential Giemsa staining of sister chromatids and study of sister chromatid exchanges without
autoradiography. Chromosoma 1974;48:341-53. 
8 ONCOR. Chromosome in situ system, edition 3, Gaithersburg, 1991 .

9 Cooke HJ, Brown WRA, Rappold GA. Hypervariable telomeric sequences from the human sex chromosomes are pseudoautosomal. Nature 1985;317:687-92.

10 Henke A, Fischer C, Rappold GA. Genetic map of the human pseudoautosomal region reveals a high rate of recombination in female meiosis at the $\mathrm{Xp}$ telomere. Genomics 1993;18:478-85.

11 Petit C, Levilliers J, Weissenbach J. Physical mapping of the human pseudo-autosomal region: comparison with genetic linkage map. $E M B O \nexists 1988 ; 8: 2369-76$.

$12 \mathrm{Lau} \mathrm{YF,} \mathrm{Kan} \mathrm{YW.} \mathrm{Direct} \mathrm{isolation} \mathrm{of} \mathrm{the} \mathrm{functional} \mathrm{human}$ thymidine kinase gene with a cosmid shuttle vector. Proc thymidine kinase gene with a cosmid
Natl Acad Sci USA 1984;81:414-18.
13 Curry JR, Magenis RE, Brown M, et al. Inherited chondrodysplasia punctata due to a deletion of the terminal short arm of an X chromosome. NEngl F Med 1984;311:1010-15.

14 Geekens C, Just W, Vogel W. Deletion of Xq and growth deficit: a review. Am $\mathcal{F}$ Med Genet 1994;50:105-13.

15 Ogata T, Matsuo N. Sex chromosome aberrations and stature: deduction of the principal factors involved in the determination of the adult height. Hum Genet 1993;91: 551-62.

16 Gilbert EF, Opitz JM. Developmental and other pathologic changes in syndromes caused by chromosome abnormalities. Perspect Pediatr Pathol 1982;7:1-63.

17 Gartler SM, Sparkes RS. The Lyon-Beutler hypothesis and isochromosome X patients with the Turner syndrome.
Lancet 1963;ii:411. 